\title{
Coupling model for an extended-range plasmonic optical transformer scanning probe
}

\author{
This article has been corrected since online publication and an erratum has also been published
}

\author{
Aleksandr Polyakov, Mauro Melli, Giuseppe Cantarella, Adam Schwartzberg, Alexander Weber-Bargioni, \\ P James Schuck and Stefano Cabrini
}

The expansion of nanoscale optics has generated a variety of scanning probe geometries that yield spatial resolution below 10 nm. In this work, we present a physical model for coupling far-field radiation to plasmonic modes on the surface of a scanning probe, and propose a scheme for extending the working distance of such a probe. In a subsurface application, an optical transformer at the tip of a probe can be coupled to a remote near-field antenna placed inside the sample at a distance away from the surface, expanding the effective working distance up to $100 \mathrm{~nm}$.

Light: Science \& Applications (2014) 3, e195; doi:10.1038/lsa.2014.76; published online 26 September 2014

Keywords: optical transformer; plasmonic tip; plasmon coupling

\section{INTRODUCTION}

Motivated by biological imaging applications, many optical schemes have been explored for increasing the spatial resolution beyond the diffraction limit. ${ }^{1,2}$ These techniques can be generally considered to be 'band limited', ${ }^{3}$ meaning that only the propagating wave vectors are collected by the imaging system. One approach to collect the information contained in the evanescent fields is to introduce a probe in the near-field of the sample. ${ }^{4,5}$ In this approach, converting far-field radiation to near-field, and vice vers $a^{3,6,7}$ is accomplished by the probe itself. In this scheme, the maximum resolution is determined by the characteristic size of the probe tip, ${ }^{8}$ which can be smaller than the diffraction limit for high resolution imaging. Such surface techniques have been demonstrated to provide high spatiotemporal resolution ${ }^{9}$ at a working distance of only a few nanometers. ${ }^{10-12}$

Increasing the working distance of such a system is a key limitation for subsurface imaging. ${ }^{13}$ One approach is a superlens ${ }^{14}$ that uses negative refraction to achieve, in principle, a perfect imaging system that retains all wave vectors of the source. Another related approach is evanescent wave amplification, where planar structures induce convergence in the near field. ${ }^{15}$ In this work, we propose a theoretical concept where the system working distance is increased by combing a near-field scanning probe with a nano-antenna within the sample. Using a scanning probe that incorporates an optical transformer at the tip, this nano-atenna can be optically coupled to the probe enabling near-field signal collection from a point within the sample and then exporting this information to the probe with a subdiffraction spatial resolution reaching $\lambda / 10$ in the near-infrared (NIR) spectral region (for all discussions that follow, $\lambda$ refers to the light wavelength in vacuum). This probe is especially suited for bio-imaging applications, such as single-cell endoscopy, ${ }^{16}$ fluorescence spectroscopy ${ }^{17}$ and other chemical mapping studies. ${ }^{18}$

A number of a scanning probes that use plasmonic nanostructures at the tip have been designed that yield superior signal collection enabling hyperspectral high resolution imaging. ${ }^{19-21}$ One such tip is based on the concept of an optical transformer $(\mathrm{OT})^{22,23}$ - a geometrical device for converting photonic to plasmonic modes-over a large bandwidth in the NIR, achieving strong field enhancement ${ }^{24}$ with low background noise. ${ }^{25}$ These advantageous properties are inherent to the design of the probe, where excitation of the sample and signal collection are achieved by the same tip. The excitation radiation is injected at the base of the probe and then converted into surface plasmon polariton (SPP) modes that undergo an adiabatic compres$\operatorname{sion}^{26}$ at the apex of the structure and couple to the sample. The collected signal undergoes the same coupling chain in the reverse order for detection. The working range of a plasmonic probe can potentially be extended beyond $100 \mathrm{~nm}$ by forming an optical link between the OT tip and the nano-antenna embedded in the sample, which serves as a remote near-field probe. First, we describe a physical model for photon to SPP coupling in the OT and determine the geometrical constraints on the spectral response. Second, we show a numerical study demonstrating the extended working distance of the probe for subsurface imaging applications.

\section{MATERIALS AND METHODS}

The OT has a unique optical response that, unlike common plasmonic resonant structures, such as bowties, ${ }^{11}$ contains both the resonant field enhancement response as well as the non-resonant field enhancement 
a

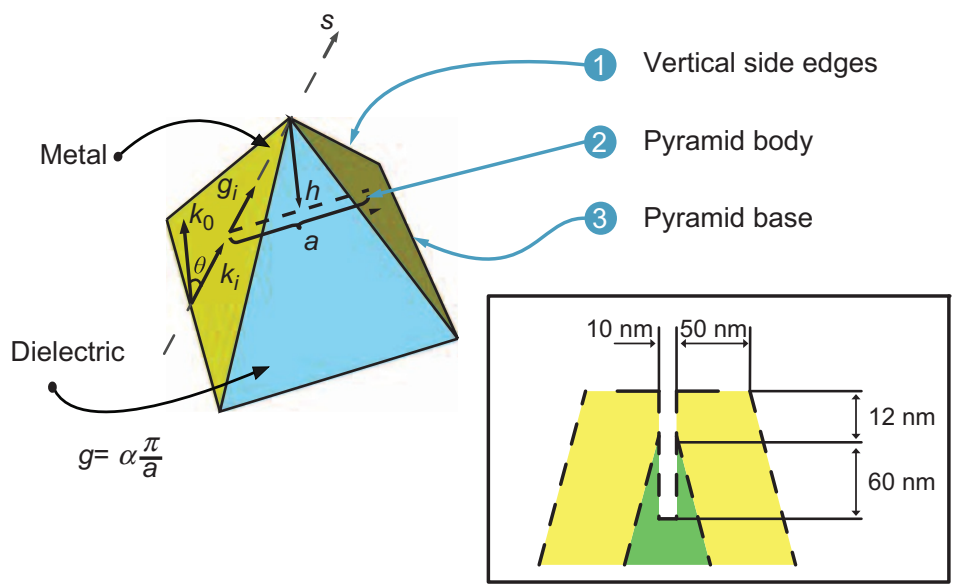

b

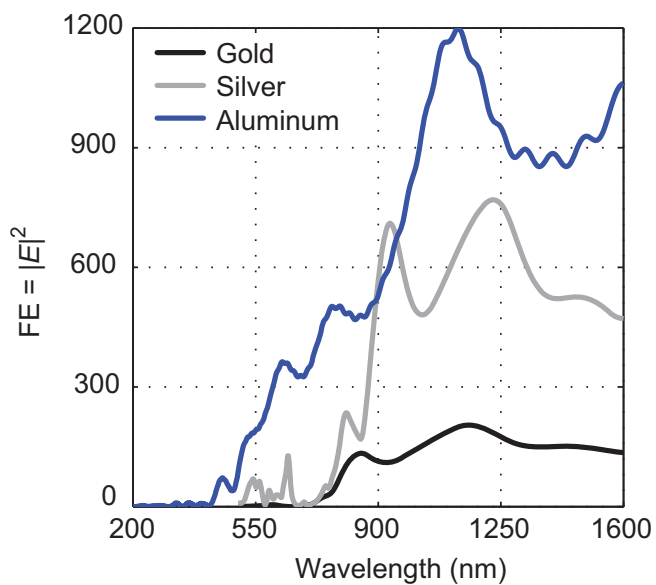

Figure 1 A scanning probe converts the far-field radiation into the near-field thereby achieving a subdiffraction spatial resolution. By incorporating a plasmonic structure (a), called the optical transformer, at the tip of the probe, the signal collection is greatly enhanced with the spatial resolution determined by the size of the gap (see inset) at the apex of the pyramid. An advantage of such a probe is its broadband response in the FE; NIR shown in (b) for a range of metals. In this geometry, the pyramid — made from a dielectric —is covered on two opposite sides by a thin metallic layer. FE, field enhancement; NIR, near infrared.

due to the adiabatic compression at the apex. We begin by exploring the non-resonant response. Figure la schematically shows the OT realized in a pyramidal structure made from a dielectric core with two opposite sides covered by a thin layer of a plasmonic material, such as a noble metal. For this theoretical study, we chose practical structure dimensions ${ }^{27}$ to explore the optical response of a realistic OT that has been achieved in practice. ${ }^{21}$

The optical response was modeled by finite difference time domain simulation (FDTD), with the structure illuminated from the bottom by light with a wave vector $\boldsymbol{k}_{\boldsymbol{0}}$. As light enters the pyramid, it is converted to the insulator-metal-insulator (IMI) SPP mode propagating along the metal-coated sides. During the approach to the apex, the IMI SPP modes on the opposite sides couple, undergoing adiabatic compression that results in strong energy localization ${ }^{28,29}$ within an ultra-small mode volume of the $10 \mathrm{~nm}$ gap cut at the apex (see inset of Figure 1a). The resulting field enhancement $(\mathrm{FE})=\left|E / E_{0}\right|^{2}$ (Figure $1 \mathrm{~b}$ ) was calculated using a field probe placed $3 \mathrm{~nm}$ directly above the gap at the apex of the OT (full simulation details are provided in the Supplementary Information). The OT spectrum has a characteristic FE cutoff in the visible and a 'rough plateau' in the NIR as shown in Figure 1b. This broadband FE response in the NIR suggests that the photon-SPP coupling mechanism is relatively wavelength independent and makes this type of a probe especially useful for spectroscopy. ${ }^{30}$

\section{RESULTS AND DISCUSSION}

The coupling between the photonic and plasmonic modes occurs within the body of the pyramid, far away from the apex, as all the photonic modes cut off due to the diffraction limit, namely, $a<\lambda / 2 n$, where $n$ is the refractive index of the dielectric core of the pyramid and $a$ is the lateral edge dimension of the pyramid. Converting light into SPP waves requires momentum matching, ${ }^{31}$ which can be accomplished at three places (refer to Figure 1a): (i) along the side edges; (ii) along the body of the pyramid; and (iii) at the base of the pyramid. Coupling along the edges is inefficient, because the SPPs propagating along these edges will also tend to outcouple by the same edges. Therefore, the spectral response of the OT is dominated by the other two coupling schemes.
In principle, an MIM waveguide has no cut off, ${ }^{32,33}$ however, when realized as a pyramid, such a structure does have a cut off due to its three-dimensional tapering: as a decreases towards the apex, the photonic modes cutoff at $a_{\min } \approx \lambda_{0} / 2 n$. This cut off becomes crucial in choosing the geometry to access shorter wavelengths and is determined primarily by the geometry of the OT and not the plasmonic properties of the materials used. For larger $a$, corresponding to a longer distance $h$ from the apex as shown in Figure 1, the rectangular waveguide geometry of the pyramid produces a transverse momentum $g=\alpha \pi / a$ ( $\alpha$ is a fitting parameter that depends on the metal optical constants and the layer thickness). This momentum contributes to coupling between the incident light and the SPP modes propagating along $s$-axis on the metallic side

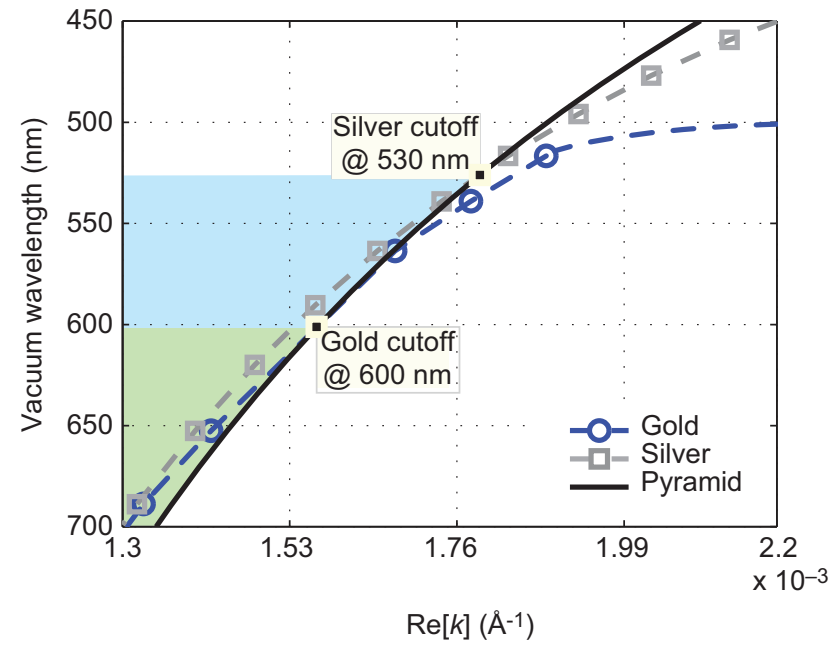

Figure 2 The pyramidal structure provides an additional momentum for converting photons to plasmon modes. The free-space dispersion for silver and gold (dashed lines) is plotted along with the maximum cumulative photon momentum inside the pyramid (solid line). The intersection of the curves corresponds to the short-wavelength cutoff for plasmon coupling in the pyramid. The shaded regions indicate all available momenta for coupling. These large regions give rise to the broadband optical response of the OT. OT, optical transformer. 


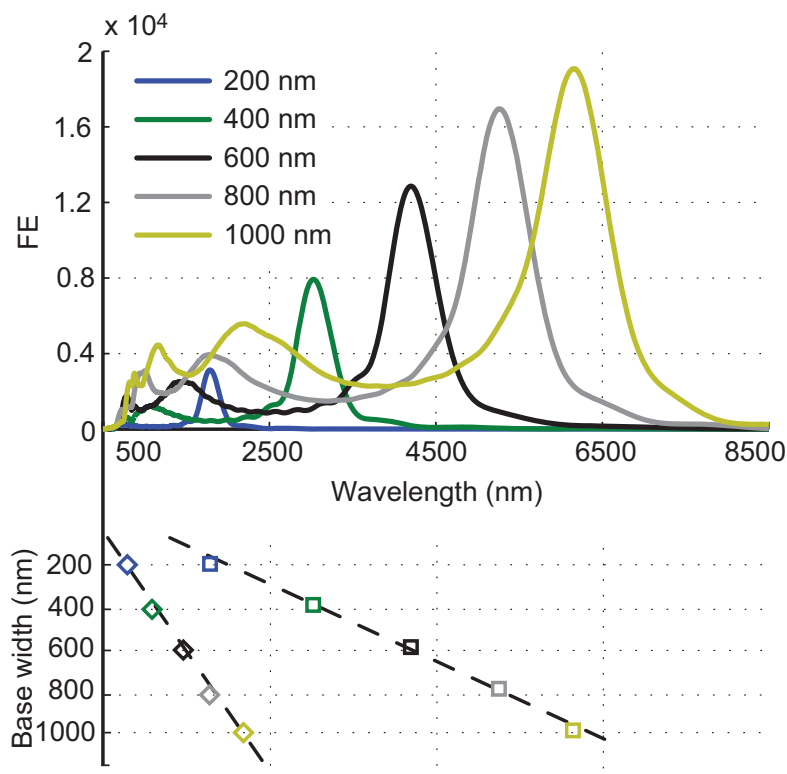

Figure 3 At longer IR wavelengths, light scatters from the very base of the pyramid launching long-range SPPs. As these waves bounce off the pyramid apex they form a standing wave along the metallic sides. In the optical spectrum obtained from the FDTD simulation, this effect is seen as sharp resonances (the fundamental and the first harmonic modes are plotted at the bottom) with a linear dependence on the width of the pyramid base. FDTD, finite difference time domain; FE, field enhancement; IR, infrared; SPP, surface plasmon polariton.

(Figure 1), similar to a grating-coupled system. ${ }^{34}$ The $s$-projection of the incident wave vector $\boldsymbol{k}_{\boldsymbol{0}}$ is added to the $s$-projection of the $\boldsymbol{g}$ wave vector to satisfy the momentum matching condition. The total sum varies depending on the distance $h$ away from the apex, with the maximum sum wave vector (corresponding to $a=\lambda_{0} / 2$ ) given by:

$$
k_{\mathrm{pyr}}=n \boldsymbol{k}_{\mathbf{0}}(\alpha \sin \theta+\cos \theta)
$$

Figure 2 shows the IMI dispersion relation for a $50 \mathrm{~nm}$ metallic film for silver and gold ${ }^{35}$ surrounded by $n=1.4$ dielectric. The dispersion relation for the combined wave vector in the pyramid $(\alpha \approx 0.3)$ is plotted with shaded regions indicating where the plasmon coupling is efficient within the OT. The intersection point of the pyramid dispersion curve with the IMI curve corresponds to the low wavelength cutoff due to the geometry of the OT. Although the material properties allow SPP coupling to extend further into the ultraviolet ${ }^{36}$ (for aluminium, the SPP limit is at $120 \mathrm{~nm}$ ), the geometrical shape of the OT ultimately determines the actual short wavelength cutoff. As the photonic mode is propagating into the pyramid, at a certain point, it encounters a matching $k_{\text {pyr }}$ wave vector needed for coupling. The result is a broadband coupling assisted by the full spectral range of available $g$ wave vectors at various distances from the apex.

In the regime where the light wavelength is longer than the base width of the pyramid, the edges at the base scatter the incident light producing large $\boldsymbol{k}$-vectors required for momentum matching. ${ }^{37}$ This process results in the SPP waves launching from the base that propagate towards the apex. However, at these long infrared (IR) wavelengths, the SPP mode is spread over a larger volume and they undergo a non-adiabatic compression ${ }^{23}$ resulting in reflection from the apex rather than adiabatic compression. The result is the formation of an SPP standing wave along the $s$-axis, where the metallic side of the OT acts as a plasmonic Fabry-Perot cavity. Figure 3 shows the full spectrum of the OT extending to the IR, where these long wavelength resonances are shown to be linearly related to the base width of the pyramid. As the base width increases, so does the overall collection efficiency of the OT resulting in the increased FE at the plateau (Supplementary Fig. S1b).

Optimizing the geometry to maximize the width and the height of the plateau region will deliver the most amount of light to the apex and thereby to the sample. For collection, incorporating a $10 \mathrm{~nm}$ gap at the apex of the pyramid dramatically improves the near-field coupling resulting in a large increase in signal collection (Supplementary Fig. S2). However, the working distance still remains limited to a few nanometers above the apex. Expanding the working distance can be a

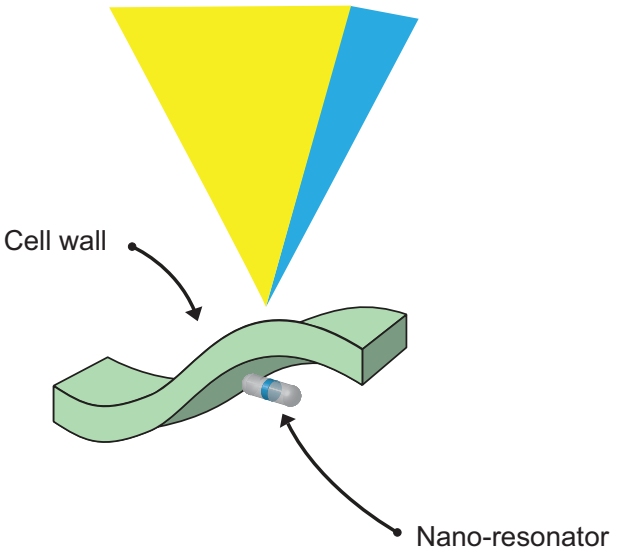

b

Long-range coupling in OT

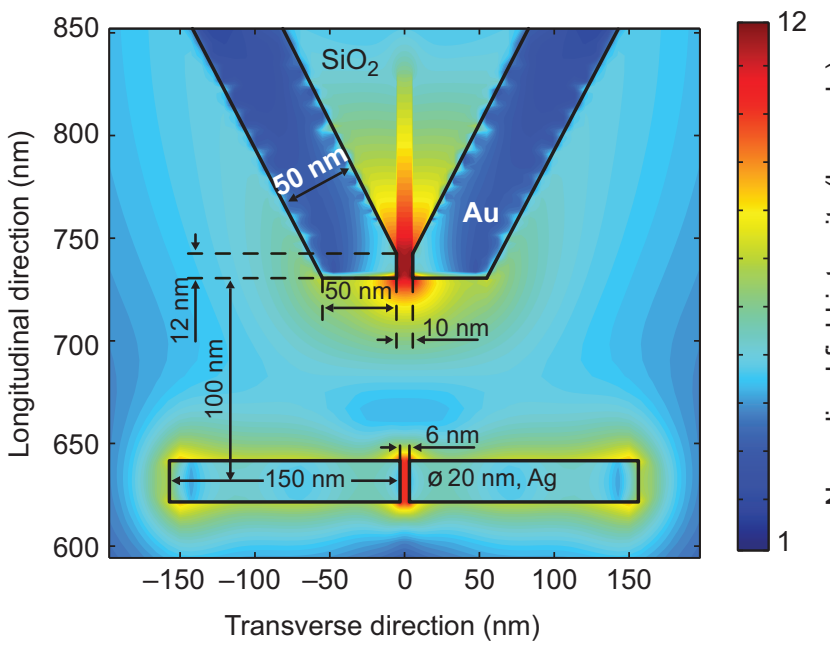

Figure 4 The effective working distance of a near-field probe can extended beyond the typical $3 \mathrm{~nm}$ by placing a resonator some distance away from the apex of the OT (a). In this configuration, the electric field couples between the probe and the resonator (b) establishing an efficient channel for locally exciting the sample at the location of the resonator hotspot and collecting the resulting signal from it. OT, optical transformer. 


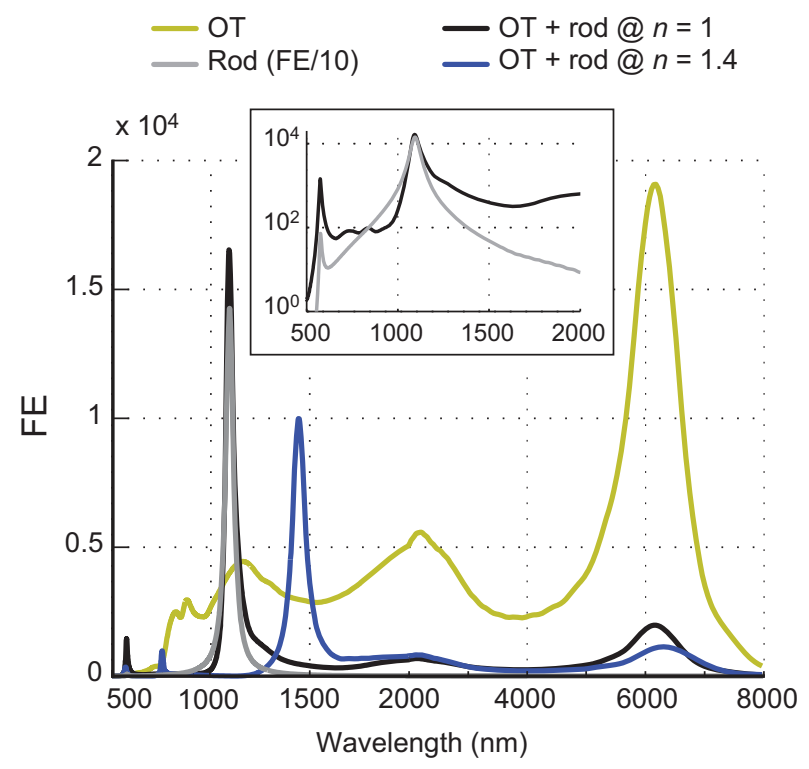

Figure 5 The FE of the NP has a single resonance at $1200 \mathrm{~nm}$ (shown as FE/10); in comparison, the OT FE spectrum has the plateau region and the IR resonance. In combination, the FE measured at the center of the NP shows the presence of features from both components (the plateau region and the resonance peak are shown in the inset) indicating the optical coupling between the OT and the NP. Placing the NP into higher refractive index medium results in a minor wavelength shift of the resonance, retaining the coupling that enables the extended working distance of the OT+NP probe. FE, field enhancement; IR, infrared; NP, nanoparticle; OT, optical transformer. accomplished by placing a nanoantenna label, such as a plasmonic nanoparticle (NP) a short distance away from the apex. For example, a metallic NP $100 \mathrm{~nm}$ away from the tip of the probe will collect and otherwise rapidly diverging the near-field radiation. This way, the NP becomes a remote near-field antenna extending the working distance of the system. Similar to the cascading plasmonic coupling, ${ }^{38}$ the radiation is compressed from the far-field to the apex of the OT at the tip of the probe, then transferred to the NP, and then to the sample in the immediate vicinity of the NP. The power of this scheme is that it works as well in delivering localized fields to the sample as it does in collecting the resulting signal from the sample, with an advantage of an extended working distance and subdiffraction spatial resolution.

This extended probe design is summarized in Figure 4a: the OT probe is used to scan across a sample such as a cell loaded with NPs. ${ }^{39}$ As the probe approaches an NP directly across the membrane the fields from the apex of the tip couple to the NP exciting the sample locally within a nm distance from the NP hotspot. The exact shape, size, and material of the NP can be engineered for a particular study to produce a hotspot at the desired wavelength. ${ }^{40}$ In this case study, the $\mathrm{NP}$ is a $300 \mathrm{~nm}$ long silver nanorod resonant around $1200 \mathrm{~nm}$ with a $6 \mathrm{~nm}$ dielectric $(n=1.4)$ spacer at its center. Although such an NP is much too large for a biological application, it is useful in demonstrating the extended-range coupling concept. The spacer concentrates the fields in a small mode volume greatly increasing the local field strength. Effectively, this type of an NP recompresses the diverging fields from the tip of the OT back into a sub-10 nm spot.

The performance of the OT + NP probe can be evaluated in terms of the total field enhancement at the NP, the spatial resolution and the a Spatial resolution in terms of NP FE

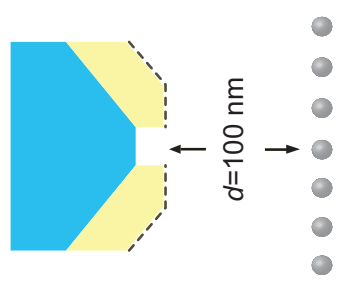

$\varnothing 20 \mathrm{~nm}$, silver NPs spaced $60 \mathrm{~nm}$ apart b
FE map of the NP matrix

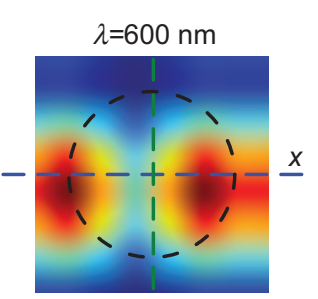

y I

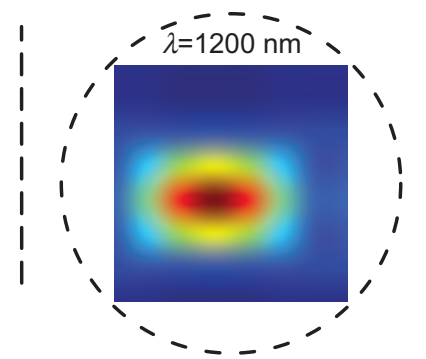

d $\quad$ Spatial resolution and FE of the OT+NP

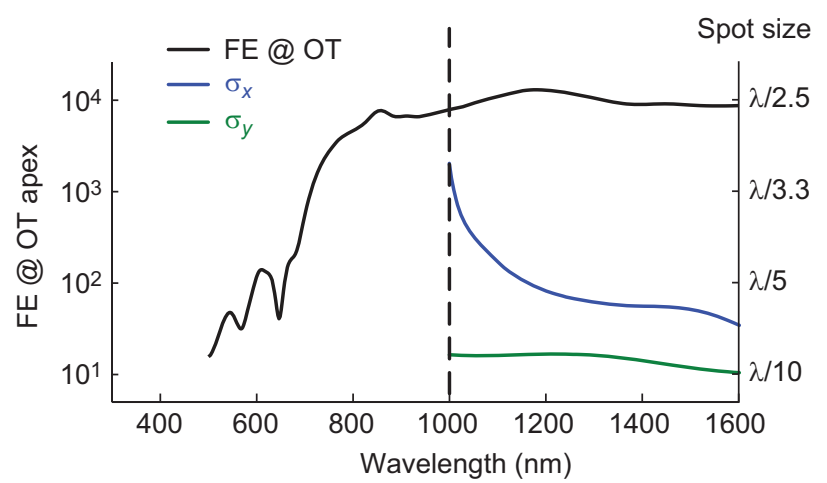

c

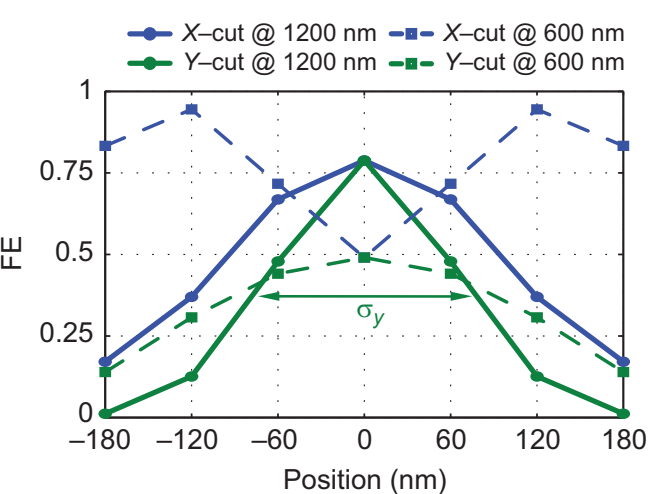

Effective focus spot size

Position (nm)

Figure 6 The spatial resolution of the OT+NP probe is sampled by measuring the FE across the NPs placed on a grid $100 \mathrm{~nm}$ away from the OT apex (a). Comparing the FE maps at $600 \mathrm{~nm}$ to $1200 \mathrm{~nm}$ shows the subdiffraction focus formation at longer wavelengths (b) due to efficient SPP coupling in the OT. The diffraction-limited spot size is indicated by a dashed circle outline. The $x$-and $y$-cuts of the FE map illustrate the spot size (c), with the width of the peak representing the spatial resolution of the OT+NP probe reaching as low as $\lambda / 10$ for NIR wavelengths (d). FE, field enhancement; NP, nanoparticle; OT, optical transformer; SPP, surface plasmon polariton. 
overall optical system complexity. The FE for the OT immediately at the outer metal surface is shown in Figure 5 in comparison to the $\mathrm{FE}=10$ in the dielectric spacer of the NP illuminated by a diffraction limited white light without the OT. Bringing the NP within $100 \mathrm{~nm}$ of the apex of the OT results in an FE spectral response that contains both: the resonance of the NP itself plus the resonances of the OT. The fields from the apex of the OT efficiently couple to the NP thereby extending the working distance of the system at the cost of overall reduced FE.

Placing this NP inside a layer of a higher refractive index medium $(n=1.4)$ produces a red spectral shift for the on-resonance response, as is expected for a plasmonic system. ${ }^{41}$ In both cases, however, the nonresonant plateau response is retained in the NIR region from 600 to $1000 \mathrm{~nm}$, as seen in the inset of Figure 5. The IR resonance of the pyramid remains at the same position, because it is a standing wave at the metal-covered sides.

In diffraction-limited optics, the spatial resolution can be defined in terms of the size of the Airy disk. ${ }^{42}$ For the OT + NP probe; the resolution is redefined in terms of the inter-NP separation distance. That is, the spatial resolution is determined as the minimum distance between the NPs such that the signal collected from the center one is about three times larger than the signal from its nearest neighbor. To estimate the spatial resolution using finite difference time domain model, a smaller-size NP was chosen: a $10 \mathrm{~nm}$ silver nanosphere placed on a virtual grid in a transverse plane $100 \mathrm{~nm}$ away from the apex of the OT with inter-NP spacing fixed at $60 \mathrm{~nm}$ (Figure 6a). The total field intensity integrated over the NP's volume is plotted as an FE map in Figure $6 \mathrm{~b}$. This map shows the effect of SPP coupling in the OT: shorter wavelengths (outside the working range of the OT) that do not get localized to the apex of the probe leak out from the sides producing two lobes, rather than a single focus. In this case, the sample is excited at every NP and the signal is also collected from every NP with a spatial resolution worse than a conventional microscope (shown by a dashed circular outline). In the NIR spectral range, however, the OT $+\mathrm{NP}$ probe produces a subdiffraction focus shown in Figure 6c.

The spatial resolution for the OT +NP probe is defined as the width of the peak $\sigma$ in the FE map across the NPs placed a distance away as in Figure 6. Due to the extent of the metal-covered sides in the $x$-direction, the NPs tend to couple to the edges of the OT resulting in $\sigma_{x}>\sigma_{y}$. For $\lambda>1200 \mathrm{~nm}$ light is compressed to the apex and from there it only excites the NPs directly across from the tip. For these longer wavelengths, the signal from the centermost NP will be predominantly collected by the OT, yielding a high spatial resolution up to $\lambda / 10$.

\section{CONCLUSIONS}

Combining an OT and a cloud of NPs in the sample can be effectively used as a new type of a probe for subsurface imaging and spectroscopy that trades high field enhancement for an increase in spatial resolution $^{3}$ at an extended working distance. By including a resonant NP reporter antenna within the sample, the weak fields extending from the probe are amplified by orders of magnitude in a nanoscale mode volume allowing for a high sensitivity measurement. Furthermore, the OT probe provides the subdiffraction resolution for both excitation and signal collection. Incorporating the OT on a flexible probe, such as an optical fiber, will make the OT $+\mathrm{NP}$ probe particularly useful for applications such as single-cell endoscopy ${ }^{16}$ and other bio-imaging applications. ${ }^{43}$

In summary, we have presented a coupling model for the broadband spectral response of an OT probe. A new scheme for extending the range of a near-field probe has been theoretically studied showing a potential for a 10 -fold increase in the working distance. Such an extended range probe that retains the subdiffraction spatial resolution and strong field enhancement without the use of a complex optical system is well suited for high-resolution subsurface imaging applications.

\section{ACKNOWLEDGMENTS}

This work was performed at the Molecular Foundry, Lawrence Berkeley National Laboratory, and was supported by the Office of Science, Office of Basic Energy Sciences, Scientific User Facilities Division of the US Department of Energy under Contract No. DE-AC02-05CH11231.

1 Schermelleh L, Heintzmann R, Leonhardt $\mathrm{H}$. A guide to superresolution fluorescence microscopy. J Cell Biol 2010; 190: 165-175.

2 Moerner WE. Microscopy beyond the diffraction limit using actively controlled single molecules. J Microsc 2012; 246: 213-220.

3 Gordon R. Limits for superfocusing with infinite evanescent wave amplification. Opt Lett 2012; 37: 912-914.

4 Novotny L, Hecht B. Principles of Nano-optics. Cambridge: Cambridge University Press; 2006.

5 Schuck PJ, Weber-Bargioni A, Ashby PD, Ogletree DF, Schwartzberg A et al. Life beyond diffraction: opening new routes to materials characterization with nextgeneration optical near-field approaches. Adv Funct Mater 2013; 23: 2539-2553.

6 Gramotnev DK, Bozhevolnyi SI. Plasmonics beyond the diffraction limit. Nat Photonics 2010; 4: 83-91.

7 Stockman MI. Nanofocusing of optical energy in tapered plasmonic waveguides. Phys Rev Lett 2004; 93: 137404.

8 Bouhelier A, Renger J, Beversluis MR, Novotny L. Plasmon-coupled tip-enhanced near-field optical microscopy. J Microsc 2003; 210: 220-224.

9 Berweger S, Atkin JM, Olmon RL, Raschke MB. Light on the tip of a needle: plasmonic nanofocusing for spectroscopy on the nanoscale. J Phys Chem Lett 2012; 3: 945952.

10 Weber-Bargioni A, Schwartzberg A, Cornaglia M, Ismach A, Urban JJ et al. Hyperspectral nanoscale imaging on dielectric substrates with coaxial optical antenna scan probes. Nano Lett 2011; 11: 1201-1207.

11 Schuck PJ, Fromm DP, Sundaramurthy A, Kino GS, Moerner WE. Improving the mismatch between light and nanoscale objects with gold bowtie nanoantennas. Phys Rev Lett 2005; 94: 017402.

12 Yu Z, Fan S, Avlasevich Y, Mullen K, Moerner WE. Large single-molecule fluorescence enhancements produced by a bowtie nanoantenna. Nat Photonics 2009; 3: 654-657.

13 Engelhardt AP, Hauer B, Taubner T. Visibility of weak contrasts in subsurface scattering near-field microscopy. Ultramicroscopy 2013; 126: 40-43.

14 Pendry JB. Negative refraction makes a perfect lens. Phys Rev Lett 2000; 85: 3966 3969.

15 Merlin R. Radiationless electromagnetic interference: evanescent-field lenses and perfect focusing. Science 2007; 317: 927-929.

16 Yan R, Park JH, Choi Y, Heo CJ, Yang SM et al. Nanowire-based single-cell endoscopy. Nat Nanotechnol 2012; 7: 191-196.

17 Liebermann T, Knoll W. Surface-plasmon field-enhanced fluorescence spectroscopy. Colloid Surf A 2000; 171: 115-130.

18 De Angelis F, Das G, Candeloro P, Patrini M, Galli M et al. Nanoscale chemical mapping using three-dimensional adiabatic compression of surface plasmon polaritons. Nat Nanotechnol 2010; 5: 67-72.

19 Olmon RL, Raschke MB. Antenna-load interactions at optical frequencies: impedance matching to quantum systems. Nanotechnology 2012; 23: 444001.

20 Ropers C, Neacsu CC, Elsaesser T, Albrecht M, Raschke MB et al. Grating-coupling of surface plasmons onto metallic tips: a nanoconfined light source. Nano Lett 2007; 7: 2784-2788.

21 Bao W, Melli M, Caselli N, Riboli F, Wiersma DS et al. Mapping local charge recombination heterogeneity by multidimensional nanospectroscopic imaging. Science 2012; 338: 1317-1321.

22 Choo H, Stafarroni M, Seok TJ, Bokor J, Wu M et al. Three-dimensional optical transformer-highly efficient nanofocusing device. In: Proceedings of Conference on Lasers and Electro-Optics 2010; 16-21 May 2010; San Jose, CA, USA. IEEE: Piscataway, NJ, USA, 2010.

23 Pile DF, Gramotnev DK. Adiabatic and nonadiabatic nanofocusing of plasmons by tapered plasmon waveguides. App/ Phys Lett 2006; 89: 041111

24 Choo H, Kim MK, Staffaroni M, Seok TJ, Bokor J et al. Nanofocusing in a metalinsulator-metal gap plasmon waveguide with a three-dimensional linear taper. Nat Photonics 2012; 6: 838-844.

25 Bao W, Staffaroni M, Bokor J, Salmeron MB, Yablonovitch E et al. Plasmonic nearfield probes: a comparison of the campanile geometry with other sharp tips. Opt Express 2013; 21: 8166-8176.

26 Gramotnev DK. Adiabatic nanofocusing of plasmons by sharp metallic grooves: geometrical optics approach. J Appl Phys 2005; 98: 104302.

27 Melli M, Polyakov A, Gargas D, Huynh C, Scipioni L et al. Reaching the theoretica resonance quality factor limit in coaxial plasmonic nano resonators fabricated by helium ion lithography. Nano Lett 2013; 13: 2687-2691. 
28 Zhou W, Dridi M, Suh JY, Kim CH, Co DT et al. Lasing action in strongly coupled plasmonic nanocavity arrays. Nat Nanotechnol 2013; 8: 506-511.

29 Lindquist NC, Johnson TW, Nagpal P, Norris DJ, Oh SH. Plasmonic nanofocusing with a metallic pyramid and an integrated C-shaped aperture. Sci Rep. 2013; 3: 1857.

30 Mivelle M, van Zanten TS, Neumann L, van Hulst NF, Garcia-Parajo MF. Ultrabright bowtie nanoaperture antenna probes studied by single molecule fluorescence. Nano Lett 2012; 12: 5972-5978.

31 Raether H. Surface Plasmons on Smooth and Rough Surfaces and on Gratings. Berlin/ Heidelberg/New York: Springer-Verlag; 1988.

32 Economou EN. Surface plasmons in thin films. Phys Rev 1969; 182: 539-554.

33 Dionne JA, Sweatlock LA, Atwater HA, Polman A. Planar metal plasmon waveguides: frequency-dependent dispersion, propagation, localization, and loss beyond the free electron model. Phys Rev B 2005; 72: 075405.

34 Polyakov A, Zolotorev M, Schuck PJ, Padmore HA. Collective behavior of impedance matched plasmonic nanocavities. Opt Express 2012; 20: 7685-7693.

35 Johnson PB, Christy RW. Optical constants of the noble metals. Phys Rev B 1972; 6 : 4370-4379.

36 Polyakov A, Cabrini S, Dhuey S, Harteneck B, Schuck PJ et al. Plasmonic light trapping in nanostructured metal surfaces. App/ Phys Lett 2011; 98: 203104.

37 Zhang L, Kubo A, Wang L, Petek H, Seideman T. Imaging of surface plasmon polariton fields excited at a nanometer-scale slit. Phys Rev B 2011; 84: 245442.

38 Toroghi S, Kik PG. Cascaded plasmon resonant field enhancement in nanoparticle dimers in the point dipole limit. Appl Phys Lett 2012; 100: 183105.
39 Kang B, Austin LA, El-Sayed MA. Real-time molecular imaging throughout the entire cell cycle by targeted plasmonic-enhanced Rayleigh/Raman spectroscopy. Nano Lett 2012; 12: 5369-5375.

40 Khlebtsov NG, Dykman LA. Optical properties and biomedical applications of plasmonic nanoparticles. J Quant Spectrosc Radiat Transfer 2010; 111: 1-35.

41 Polyakov A, Thompson KF, Dhuey SD, Olynick DL, Cabrini S et al. Plasmon resonance tuning in metallic nanocavities. Sci Rep 2012; 2: 933.

42 Airy GB. On the diffraction of an object-glass with circular aperture. Trans Cambridge Philos Soc 1835; 5: 283-291.

43 Anker JN, Hall WP, Lyandres O, Shah NC, Zhao J et al. Biosensing with plasmonic nanosensors. Nat Mater 2008; 7: 442-453.

cc)(i) (2) This work is licensed under a Creative Commons Attribution-

c. NG SA NonCommercial-ShareAlike 3.0 Unported License. The images or other third party material in this article are included in the article's Creative Commons license, unless indicated otherwise in the credit line; if the material is not included under the Creative

Commons license, users will need to obtain permission from the license holder to reproduce the material. To view a copy of this license, visit http://creativecommons.org/licenses/ by-nc-sa/3.0/ 